\title{
Lucas polynomial solution of nonlinear differential equations with variable delays
}

\author{
Sevin Gümgüm*1 (D), Nurcan Baykuş Savaşaneril ${ }^{2}$ (D), Ömür Kıvanç Kürkçü ${ }^{1}$ (D), \\ Mehmet Sezer $^{3}$ (1) \\ ${ }^{1}$ Izmir University of Economics, Department of Mathematics, Izmir, 35330, Turkey \\ ${ }^{2}$ Izmir Vocational School, Dokuz Eylül University, Izmir, Turkey \\ ${ }^{3}$ Department of Mathematics, Manisa Celal Bayar University, Manisa, Turkey
}

\begin{abstract}
In this study, a novel matrix method based on Lucas series and collocation points has been used to solve nonlinear differential equations with variable delays. The application of the method converts the nonlinear equation to a matrix equation which corresponds to a system of nonlinear algebraic equations with unknown Lucas coefficients. The method is tested on three problems to show that it allows both analytical and approximate solutions.
\end{abstract}

Mathematics Subject Classification (2010). 34A34, 65L60, 40C05

Keywords. nonlinear delay differential equations, variable delays, matrix and collocation methods, Lucas polynomials and series

\section{Introduction}

Ordinary differential equations fail to model many physical phenomena when the model is not only determined by its present state but also by a certain past state. Consequently, differential equations with time delays are used in modeling of real life situations such as human body control and multibody control systems, electric circuits, dynamical behaviour of a system in fluid mechanics, chemical engineering [21], spread of bacteriophage infection [38], stage structured populations [37], epidemic model in biology and dynamic diseases model in physiology [35].

Some numerical methods have been developed to solve nonlinear differential equations (NDE) with proportional and constant delays; among them, one can mention Aboodh transformation method [5], Adomian decomposition method [12,31], Power series method [11], Decomposition method [39], Differential transform method [28], Hermite wavelet based method [36], Variational iteration method [25,29,41], Power and Padé series based method [24], Spectral method [6], Variable multistep methods [27], Quasilinearization technique [34], Runge-Kutta-Fehlberg methods [30], Polynomial least squares method [13], Homotopy perturbation method [35], and First Boubaker polynomial approach [16].

On the other hand, there are few studies about nonlinear differential equations with variable delays. A study on the existence of positive $\omega$-periodic solutions has been carried

*Corresponding Author.

Email addresses: sevin.gumgum@ieu.edu.tr (S. Gümgüm), nurcan.savasaneril@deu.edu.tr (N.B. Savaşaneril), omur.kivanc@ieu.edu.tr (Ö.K. Kürkçü), mehmet.sezer@cbu.edu.tr (M. Sezer)

Received: 18.09.2018; Accepted: 22.01.2019 
out by Dorociaková and Olach [19]. Chen et.al. [14] presented new criteria for asymptotic stability. Asymptotic behaviour of solutions is studied by Dix [18]. Fixed points and stability are studied $[7,17,23,44]$. Only a few numerical techniques have been applied to solve such kind of equations: A new multi-step technique [10], Legendre-Gauss collocation method [40] and Runge-Kutta method using Hermite interpolation [22].

Numerical solutions of ODEs, fractional differential equations (FDE) and integrodifferential equations are of great interest. Recently, methods based on Lucas, Fibonacci and Fermat polynomials have been proposed to solve FDEs $[1-4,8,42,43]$. In these studies, they derived the operational matrix of fractional derivatives and observed that the numerical solutions have smaller errors than those obtained by using orthogonal polynomials.

In the present study, we consider the NDE with variable delays of the form

$$
\sum_{k=0}^{2} \sum_{j=0}^{1} P_{k j}(t) y^{(k)}\left(t-\tau_{k j}(t)\right)+\sum_{p=0}^{1} \sum_{q=0}^{p} R_{p q}(t) y^{(p)}(t) y^{(q)}(t)=g(t)
$$

or precisely

$$
\begin{aligned}
& P_{00}(t) y\left(t-\tau_{00}(t)\right)+P_{10}(t) y^{\prime}\left(t-\tau_{10}(t)\right)+P_{01}(t) y\left(t-\tau_{01}(t)\right)+P_{11}(t) y^{\prime}\left(t-\tau_{11}(t)\right) \\
& +P_{20}(t) y^{\prime \prime}\left(t-\tau_{20}(t)\right)+P_{21}(t) y^{\prime \prime}\left(t-\tau_{21}(t)\right)+R_{00}(t) y^{2}(t)+R_{10}(t) y^{\prime}(t) y(t) \\
& +R_{11}(t)\left(y^{\prime}(t)\right)^{2}=g(t)
\end{aligned}
$$

with the initial conditions $y(a)=\lambda_{1}$ and $y^{\prime}(a)=\lambda_{2}$.

Here, $P_{k j}(t), R_{p q}(t), g(t)$, and the variable delays $\tau_{k j}(t)$ are given continuous functions defined on $0 \leq a \leq t \leq b$, where $\tau_{k j}(t) \geq 0$.

We propose a new matrix technique, developed by Sezer et.al. [9, 15, 20,32], to solve Eq. (1.1) with the initial conditions, in the finite Lucas series of the form

$$
y(t) \cong y_{N}(t)=\sum_{n=0}^{N} a_{n} L_{n}(t), \quad a \leq t \leq b
$$

where $a_{n}, n=0,1, \ldots, N$ are unknown coefficients and $L_{n}(t), n=0,1, \ldots, N ; N \geq m$ are the Lucas polynomials [26]. These polynomials are constructed from the recurrence relation

$$
\begin{aligned}
& L_{0}(t)=2, L_{1}(t)=t \\
& L_{n+2}(t)=t L_{n+1}(t)+L_{n}(t), n \geq 0
\end{aligned}
$$

The Binet and power form representations of Lucas polynomials can be seen in $[2,3]$.

\section{Operational matrix relations}

In this section, we derive the operational matrix relations of Eq. (1.1) and (1.2). For this purpose, we write the series in Eq. (1.2) as a matrix equation as follows

$$
y(t) \cong y_{N}(t)=\mathbf{L}(t) \mathbf{A}=\mathbf{T}(t) \mathbf{M A}
$$

where

$$
\begin{aligned}
& \mathbf{L}(t)=\left[\begin{array}{llll}
L_{0}(t) & L_{1}(t) & \cdots & L_{N}(t)
\end{array}\right], \quad \mathbf{T}(t)=\left[\begin{array}{llll}
1 & t & \cdots & t^{N}
\end{array}\right], \\
& \mathbf{A}=\left[\begin{array}{llll}
a_{0} & a_{1} & \cdots & a_{N}
\end{array}\right]^{T}
\end{aligned}
$$


and $\mathbf{M}$ has the form

$$
\mathbf{M}^{T}=\left[\begin{array}{ccccc}
2 & 0 & 0 & \cdots & 0 \\
0 & \frac{1}{1}\left(\begin{array}{c}
1 \\
0
\end{array}\right) & 0 & \cdots & 0 \\
\frac{2}{1}\left(\begin{array}{c}
1 \\
1
\end{array}\right) & 0 & \frac{2}{2}\left(\begin{array}{c}
2 \\
0
\end{array}\right) & & 0 \\
0 & \frac{3}{2}\left(\begin{array}{c}
2 \\
1
\end{array}\right) & 0 & \cdots & 0 \\
\vdots & \vdots & \vdots & \ddots & \vdots \\
\frac{(n-1)}{\left(\frac{n-1}{2}\right)}\left(\begin{array}{c}
\frac{n-1}{2} \\
\left.\frac{n-1}{2}\right)
\end{array}\right. & 0 & \frac{n-1}{\left(\frac{n+1}{2}\right)}\left(\frac{n+1}{\frac{n-3}{2}}\right) & \cdots & 0 \\
0 & \frac{n}{\left(\frac{n+1}{2}\right)}\left(\frac{n+1}{2}\right) & 0 & \cdots & \frac{n}{n}\left(\begin{array}{c}
n \\
0
\end{array}\right)
\end{array}\right]
$$

when $N$ is odd, and

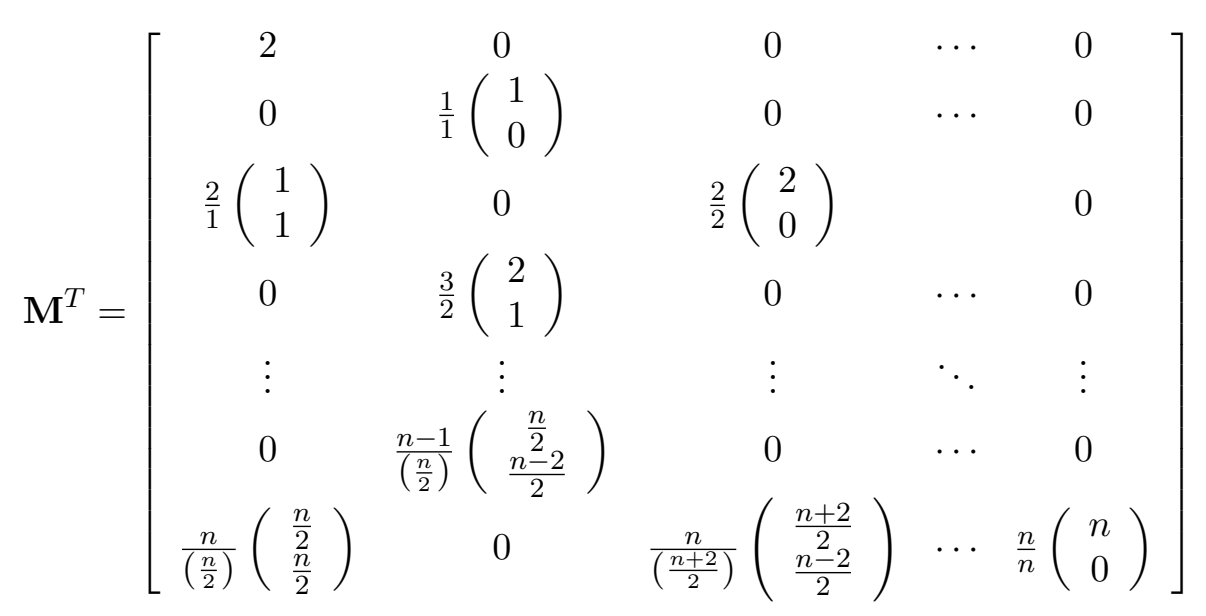

when $N$ is even.

One can write the relation between $\mathbf{T}(t)$ and its derivatives $\mathbf{T}^{\prime}(t)$ and $\mathbf{T}^{\prime \prime}(t)$ as follows

$$
\mathbf{T}^{\prime}(t)=\mathbf{T}(t) \mathbf{B} \text { and } \mathbf{T}^{\prime \prime}(t)=\mathbf{T}(t) \mathbf{B}^{2}
$$

where

$$
\mathbf{B}=\left[\begin{array}{ccccc}
0 & 1 & 0 & \cdots & 0 \\
0 & 0 & 2 & \cdots & 0 \\
\vdots & \vdots & \vdots & \ddots & \vdots \\
0 & 0 & 0 & \cdots & N \\
0 & 0 & 0 & \cdots & 0
\end{array}\right] \quad \text { and } \quad \mathbf{B}^{0}=\left[\begin{array}{ccccc}
1 & 0 & 0 & \cdots & 0 \\
0 & 1 & 0 & \cdots & 0 \\
\vdots & \vdots & \vdots & \ddots & \vdots \\
0 & 0 & 0 & 1 & 0 \\
0 & 0 & 0 & \cdots & 1
\end{array}\right]
$$

In a similar way, the approximate solution and its derivatives can be expressed by using Eq. (2.1) and (2.2) as

$$
\begin{gathered}
y(t) \cong y_{N}(t)=\mathbf{L}(t) \mathbf{A}=\mathbf{T}(t) \mathbf{M A} \\
y^{\prime}(t) \cong y_{N}^{\prime}(t)=\mathbf{T}^{\prime}(t) \mathbf{M A}=\mathbf{T}(t) \mathbf{B M A} \\
y^{\prime \prime}(t) \cong y_{N}^{\prime \prime}(t)=\mathbf{T}^{\prime \prime}(t) \mathbf{M A}=\mathbf{T}(t) \mathbf{B}^{2} \mathbf{M} \mathbf{A}
\end{gathered}
$$


Replacing $t$ by $t-\tau_{k j}(t)$ in each equation in (2.3) yields the recurrence relation,

$$
\begin{aligned}
& y\left(t-\tau_{k j}(t)\right) \cong y_{N}\left(t-\tau_{k j}(t)\right)=\mathbf{T}\left(t-\tau_{k j}(t)\right) \mathbf{M A}=\mathbf{T}(t) \mathbf{S}\left(-\tau_{k j}(t)\right) \mathbf{M A} \\
& y^{\prime}\left(t-\tau_{k j}(t)\right) \cong y_{N}^{\prime}\left(t-\tau_{k j}(t)\right)=\mathbf{T}\left(t-\tau_{k j}(t)\right) \mathbf{B} \mathbf{M A}=\mathbf{T}(t) \mathbf{S}\left(-\tau_{k j}(t)\right) \mathbf{B M A} \\
& y^{\prime \prime}\left(t-\tau_{k j}(t)\right) \cong y_{N}^{\prime \prime}\left(t-\tau_{k j}(t)\right)=\mathbf{T}\left(t-\tau_{k j}(t)\right) \mathbf{B}^{2} \mathbf{M} \mathbf{A}=\mathbf{T}(t) \mathbf{S}\left(-\tau_{k j}(t)\right) \mathbf{B}^{2} \mathbf{M A}
\end{aligned}
$$

Note that, $\mathbf{T}\left(t-\tau_{k j}(t)\right)=\mathbf{T}(t) \mathbf{S}\left(-\tau_{k j}(t)\right)$ and

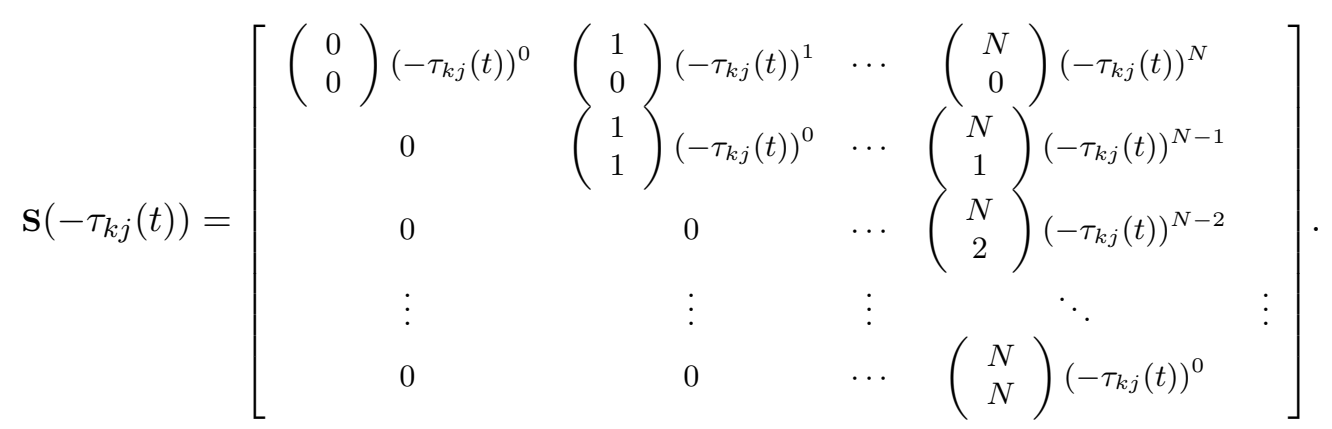

In addition, we can obtain the matrix forms of $\left(y^{(0)}(t)\right)^{2}, y^{(1)}(t) y^{(0)}(t)$ and $\left(y^{(1)}(t)\right)^{2}$ which appears in the nonlinear part of Eq. (1.1), by using Eq. (2.3) as

$$
\begin{aligned}
& \left(y^{(0)}(t)\right)^{2}=\mathbf{T}(t) \mathbf{M} \overline{\mathbf{T}(t)} \overline{\mathbf{M}} \overline{\mathbf{A}} \\
& y^{(1)}(t) y^{(0)}(t)=\mathbf{T}(t) \mathbf{B M} \overline{\mathbf{T}(t)} \overline{\mathbf{M}} \overline{\mathbf{A}} \\
& \left(y^{(1)}(t)\right)^{2}=\mathbf{T}(t) \mathbf{B M} \overline{\mathbf{T}(t)} \overline{\mathbf{B}} \overline{\mathbf{M}} \overline{\mathbf{A}}
\end{aligned}
$$

where

$$
\begin{aligned}
& \overline{\mathbf{T}}(t)=\operatorname{diag}[\mathbf{T}(t)]_{(N+1) \times(N+1)^{2}}, \quad \overline{\mathbf{M}}=\operatorname{diag}[\mathbf{M}]_{(N+1)^{2} \times(N+1)^{2}}, \\
& \overline{\mathbf{B}}=\operatorname{diag}[\mathbf{B}]_{(N+1)^{2} \times(N+1)^{2}}, \quad \overline{\mathbf{A}}=\left[\begin{array}{llll}
a_{0} \mathbf{A} & a_{1} \mathbf{A} & \cdots & a_{N} \mathbf{A}
\end{array}\right]^{T}
\end{aligned}
$$

Substituting the collocation points $\left(t_{i}=a+(b-a) i / N, i=0,1, \cdots, N\right)$ into Eq. (1.1), gives the system of equations

$$
\sum_{k=0}^{2} \sum_{j=0}^{1} P_{k j}\left(t_{i}\right) y^{(k)}\left(t_{i}-\tau_{k j}\left(t_{i}\right)\right)+\sum_{p=0}^{1} \sum_{q=0}^{p} R_{p q}\left(t_{i}\right) y^{(p)}\left(t_{i}\right) y^{(q)}\left(t_{i}\right)=g\left(t_{i}\right),
$$

which can be expressed with the aid of Eqs. (2.4) and (2.5) as

$$
\sum_{k=0}^{2} \sum_{j=0}^{1} \mathbf{P}_{k j} \overline{\mathbf{T}} \mathbf{S}_{k j} \mathbf{B}^{k} \mathbf{M A}+\sum_{p=0}^{1} \sum_{q=0}^{p} \mathbf{R}_{p q} \mathbf{T}_{p q} \overline{\mathbf{A}}=\mathbf{G}
$$


where

$$
\begin{aligned}
& \mathbf{P}_{k j}=\operatorname{diag}\left[\begin{array}{llll}
P_{k j}\left(t_{0}\right) & P_{k j}\left(t_{1}\right) & \cdots & P_{k j}\left(t_{N}\right)
\end{array}\right], \\
& \overline{\mathbf{T}}=\left[\begin{array}{c}
T\left(t_{0}\right) \\
T\left(t_{1}\right) \\
\vdots \\
T\left(t_{N}\right)
\end{array}\right]=\left[\begin{array}{cccc}
1 & t_{0} & \cdots & t_{0}^{N} \\
1 & t_{1} & \cdots & t_{1}^{N} \\
\vdots & \vdots & \ddots & \vdots \\
1 & t_{N} & \cdots & t_{N}^{N}
\end{array}\right], \\
& \mathbf{S}_{k j}=\left[\begin{array}{c}
S\left(-\tau_{k j}\left(t_{0}\right)\right) \\
S\left(-\tau_{k j}\left(t_{1}\right)\right) \\
\vdots \\
S\left(-\tau_{k j}\left(t_{N}\right)\right)
\end{array}\right], \quad \mathbf{G}=\left[\begin{array}{c}
g\left(t_{0}\right) \\
g\left(t_{1}\right) \\
\vdots \\
g\left(t_{N}\right)
\end{array}\right], \\
& \mathbf{R}_{p q}=\operatorname{diag}\left[\begin{array}{llll}
R_{p q}\left(t_{0}\right) & R_{p q}\left(t_{1}\right) & \cdots & R_{p q}\left(t_{N}\right)
\end{array}\right], \\
& \mathbf{T}_{p q}=\left[\begin{array}{c}
T\left(t_{0}\right) \mathbf{B}^{p} \overline{\mathbf{M}} \overline{T\left(t_{0}\right)} \overline{B^{q}} \overline{\mathbf{M}} \\
T\left(t_{1}\right) \mathbf{B}^{p} \overline{\mathbf{M}} \overline{T\left(t_{1}\right)} \overline{B^{q}} \overline{\mathbf{M}} \\
\vdots \\
T\left(t_{N}\right) \mathbf{B}^{p} \overline{\mathbf{M}} \overline{T\left(t_{N}\right)} \overline{B^{q}} \overline{\mathbf{M}}
\end{array}\right] ; \quad p, q=0,1 .
\end{aligned}
$$

The fundamental matrix equation (2.6) can be briefly expressed in the form

$$
\mathbf{W A}+\mathbf{Z} \overline{\mathbf{A}}=\mathbf{G}
$$

where

$$
\begin{aligned}
& \mathbf{W}=\sum_{k=0}^{2} \sum_{j=0}^{1} \mathbf{P}_{k j} \overline{\mathbf{T}} \mathbf{S}_{k j} \mathbf{B}^{k} \mathbf{M}=\left[w_{i j}\right] ; i, j=0,1, \cdots, N \\
& \mathbf{Z}=\sum_{p=0}^{1} \sum_{q=0}^{p} \mathbf{R}_{p q} \mathbf{T}_{p q}=\left[z_{m n}\right] ; \quad m=0,1, \cdots, N, \quad n=0,1, \cdots,(N+1)^{2} \\
& \mathbf{G}=\left[\begin{array}{llll}
g\left(t_{0}\right) & g\left(t_{1}\right) & \cdots & g\left(t_{N}\right)
\end{array}\right]^{T} .
\end{aligned}
$$

Also we can write the matrix equation $(2.7)$ in the augmented form as

$$
[\mathbf{W} ; \mathbf{Z} ; \mathbf{G}]=\left[\begin{array}{ccccccccccc}
w_{00} & w_{01} & \cdots & w_{0 N} & ; & z_{00} & z_{01} & \cdots & z_{0(N+1)^{2}} & ; & g\left(t_{0}\right) \\
w_{10} & w_{11} & \cdots & w_{1 N} & ; & z_{10} & z_{11} & \cdots & z_{1(N+1)^{2}} & ; & g\left(t_{1}\right) \\
\vdots & \vdots & \ddots & \vdots & ; & \vdots & \vdots & \ddots & \vdots & \ddots & \vdots \\
w_{N 0} & w_{N 1} & \cdots & w_{N N} & ; & z_{N 0} & z_{N 1} & \cdots & z_{N(N+1)^{2}} & ; & g\left(t_{N}\right)
\end{array}\right]
$$

Now, let us write the initial conditions $y(a)=\lambda_{1}$ and $y^{\prime}(a)=\lambda_{2}$ in the matrix form by using the relations in Eq. (2.3)

$$
\mathbf{T}(a) \mathbf{M A}=\boldsymbol{\lambda}_{1} \text { and } \mathbf{T}(a) \mathbf{B M A}=\boldsymbol{\lambda}_{2}
$$

or briefly

$$
\mathbf{U}_{1} \mathbf{A}+\mathbf{O}^{*} \overline{\mathbf{A}}=\boldsymbol{\lambda}_{1} \text { and } \mathbf{U}_{2} \mathbf{A}+\mathbf{O}^{*} \overline{\mathbf{A}}=\boldsymbol{\lambda}_{2}
$$

where

$$
\begin{aligned}
\mathbf{U}_{1} & =\left[\begin{array}{llll}
u_{00} & u_{01} & \cdots & u_{0 N}
\end{array}\right]=\mathbf{T}(a) \mathbf{M}, \\
\mathbf{U}_{2} & =\left[\begin{array}{llll}
u_{10} & u_{11} & \cdots & u_{1 N}
\end{array}\right]=\mathbf{T}(a) \mathbf{B M}, \\
\mathbf{O}^{*} & =\left[\begin{array}{llll}
0 & 0 & \cdots & 0
\end{array}\right] .
\end{aligned}
$$


In order to find the unknown Lucas coefficients $a_{n},(n=0,1, \cdots, N)$, related to the approximate solution Eq. (1.2), we replace the row matrices in Eq. (2.9) by any rows of the augmented matrix in Eq. (2.8). Consequently, we obtain a new augmented matrix $[\widetilde{\mathbf{W}} ; \widetilde{\mathbf{Z}} ; \widetilde{\mathbf{G}}]$ related to the matrix equation $\widetilde{\mathbf{W}} \mathbf{A}+\widetilde{\mathbf{Z}} \overline{\mathbf{A}}=\widetilde{\mathbf{G}}$.

We solve this nonlinear algebraic system using NSolve routine in Mathematica, and obtain the unknown coefficients. Then, substitute them in Eq. (2.1) to obtain the approximate solution. A detailed theoretical convergence and error analysis of Lucas expansion of a function is given in $[2,3]$. Thus, rate of convergence is investigated numerically in this study.

\section{Examples and discussion}

In this section, we apply the method to three problems to demonstrate the validity and accuracy of the method. In the first problem, the application of the method yields the exact solution. In order to show the efficiency of the method for the next two problems, we compute the absolute errors for each collocation point $t_{i}$ as follows

$$
E_{N}\left(t_{i}\right)=\left|y\left(t_{i}\right)-y_{N}\left(t_{i}\right)\right|
$$

\subsection{Example 1:}

Consider the first order nonlinear differential equation with variable delay $t^{2}$ :

$$
\left\{\begin{array}{l}
y^{\prime}(t)+t y\left(t-t^{2}\right)+t y^{2}(t)=1+t^{2}, \quad 0 \leq t \leq 1 \\
y(0)=0
\end{array}\right.
$$

The exact solution of the above problem is $y(t)=t$. We aim to show that the exact solution could be found using the present method.

First, we approximate the solution $y(t)$ by the Lucas polynomial $y_{N}(t)=\sum_{k=0}^{N} a_{k} L_{k}(t)$, and formulate the problem in the form of Eq. (1.1). Here,

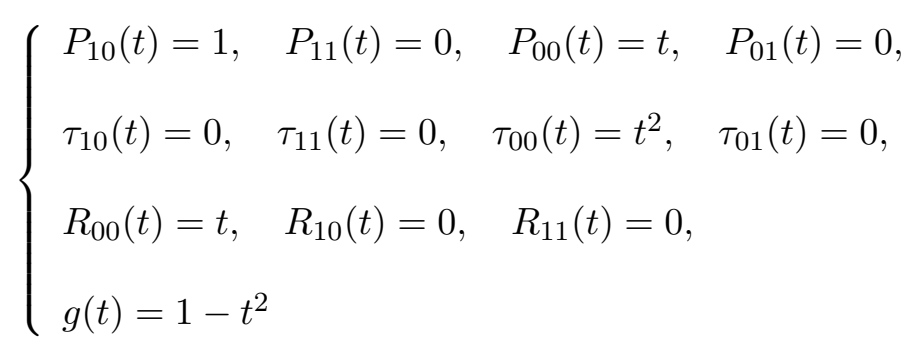

The collocation points are computed as $\left\{t_{0}=0, t_{1}=\frac{1}{2}, t_{2}=1\right\}$ by taking $N=2$. Then, we write the fundamental matrix equation of the given problem as

$$
\underbrace{\left[\mathbf{P}_{10} \overline{\mathbf{T}} \mathbf{S}_{10} \mathbf{B}^{1} \mathbf{M}+\mathbf{P}_{00} \overline{\mathbf{T}} \mathbf{S}_{00} \mathbf{B}^{0} \mathbf{M}\right]}_{\mathbf{W}} \mathbf{A}+\underbrace{\mathbf{R}_{00} \mathbf{T}_{00}}_{\mathbf{Z}} \overline{\mathbf{A}}=\mathbf{G} .
$$

where 


$$
\begin{aligned}
& \mathbf{P}_{10}=\left[\begin{array}{lll}
1 & 0 & 2 \\
0 & 1 & 0 \\
0 & 0 & 1
\end{array}\right], \quad \overline{\mathbf{T}}=\left[\begin{array}{lllllllll}
1 & 0 & 0 & 0 & 0 & 0 & 0 & 0 & 0 \\
0 & 0 & 0 & 1 & \frac{1}{2} & \frac{1}{4} & 0 & 0 & 0 \\
0 & 0 & 0 & 0 & 0 & 0 & 1 & 1 & 1
\end{array}\right], \\
& \mathbf{S}_{10}=\left[\begin{array}{lll}
1 & 0 & 0 \\
0 & 1 & 0 \\
0 & 0 & 1 \\
1 & 0 & 0 \\
0 & 1 & 0 \\
0 & 0 & 1 \\
1 & 0 & 0 \\
0 & 1 & 0 \\
0 & 0 & 1
\end{array}\right], \quad \mathbf{B}^{1}=\left[\begin{array}{lll}
0 & 1 & 0 \\
0 & 0 & 2 \\
0 & 0 & 0
\end{array}\right], \mathbf{M}=\left[\begin{array}{lll}
2 & 0 & 2 \\
0 & 1 & 0 \\
0 & 0 & 1
\end{array}\right], \\
& \mathbf{P}_{00}=\left[\begin{array}{ccc}
0 & 0 & 0 \\
0 & \frac{1}{2} & 0 \\
0 & 0 & 1
\end{array}\right], \mathbf{S}_{00}=\left[\begin{array}{ccc}
1 & 0 & 0 \\
0 & 1 & 0 \\
0 & 0 & 1 \\
0 & -\frac{1}{21} & \frac{1}{16} \\
0 & 1 & -\frac{1}{2} \\
0 & 0 & 1 \\
1 & -1 & 1 \\
0 & 1 & -1 \\
0 & 0 & 1
\end{array}\right], \mathbf{R}_{00}=\left[\begin{array}{ccc}
0 & 0 & 0 \\
0 & \frac{1}{2} & 0 \\
0 & 0 & 1
\end{array}\right], \\
& \mathbf{T}_{00}=\left[\begin{array}{ccccccccc}
4 & 0 & 4 & 0 & 0 & 0 & 4 & 2 & 6 \\
4 & 0 & 4 & 1 & \frac{1}{4} & \frac{9}{8} & \frac{9}{2} & \frac{9}{4} & \frac{27}{4} \\
4 & 0 & 4 & 2 & \frac{1}{2} & \frac{9}{4} & 6 & 3 & 9
\end{array}\right], \quad \mathbf{G}=\left[\begin{array}{c}
1 \\
\frac{5}{4} \\
2
\end{array}\right]
\end{aligned}
$$

Now, we can calculate $\mathbf{W}$ and $\mathbf{Z}$

$$
\begin{aligned}
& \mathbf{W}=\mathbf{P}_{10} \overline{\mathbf{T}} \mathbf{S}_{10} \mathbf{B}^{1} \mathbf{M}+\mathbf{P}_{00} \overline{\mathbf{T}} \mathbf{S}_{00} \mathbf{B}^{0} \mathbf{M}=\left[\begin{array}{cccc}
0 & 1 & 0 \\
1 & 1.125 & 2.03125 \\
2 & 1 & 4
\end{array}\right], \\
& \mathbf{Z}=\mathbf{R}_{00} \mathbf{T}_{00}=\left[\begin{array}{ccccccccc}
0 & 0 & 0 & 0 & 0 & 0 & 0 & 0 & 0 \\
2 & 0 & 2 & \frac{1}{2} & \frac{1}{8} & \frac{9}{16} & \frac{9}{4} & \frac{9}{8} & \frac{27}{8} \\
4 & 0 & 4 & 2 & \frac{1}{2} & \frac{9}{4} & 6 & 3 & 9
\end{array}\right] .
\end{aligned}
$$

Hence the augmented matrix $[\mathbf{W} ; \mathbf{Z} ; \mathbf{G}]$ can be written as

$$
\left[\begin{array}{ccccccccccccccc}
0 & 1 & 0 & ; & 0 & 0 & 0 & 0 & 0 & 0 & 0 & 0 & 0 & ; & 1 \\
1 & 1.125 & 2.03125 & ; & 2 & 0 & 2 & \frac{1}{2} & \frac{1}{8} & \frac{9}{16} & \frac{9}{4} & \frac{9}{8} & \frac{27}{8} & ; & \frac{5}{4} \\
2 & 1 & 4 & ; & 4 & 0 & 4 & 2 & \frac{1}{2} & \frac{9}{4} & 6 & 3 & 9 & ; & 2
\end{array}\right]
$$

The initial condition matrix is calculated as

$$
\mathbf{U}=\left[\begin{array}{lllllllllllllll}
2 & 0 & 2 & ; & 0 & 0 & 0 & 0 & 0 & 0 & 0 & 0 & 0 & ; & 0
\end{array}\right] .
$$

Replacing this row by the third row of the augmented matrix gives 


$$
\left[\begin{array}{ccccccccccccccc}
0 & 1 & 0 & ; & 0 & 0 & 0 & 0 & 0 & 0 & 0 & 0 & 0 & ; & 1 \\
1 & 1.125 & 2.03125 & ; & 2 & 0 & 2 & \frac{1}{2} & \frac{1}{8} & \frac{9}{16} & \frac{9}{4} & \frac{9}{8} & \frac{27}{8} & ; & \frac{5}{4} \\
2 & 0 & 2 & ; & 0 & 0 & 0 & 0 & 0 & 0 & 0 & 0 & 0 & ; & 0
\end{array}\right]
$$

Once we solve this system, we get the unknown coefficients as $\mathbf{A}=\left[\begin{array}{lll}0 & 1 & 0\end{array}\right]^{T}$, and hence we obtain the analytical solution

$$
y(t)=\mathbf{L}(t) \mathbf{A}=\left[\begin{array}{lll}
2 & t & t^{2}+2
\end{array}\right]\left[\begin{array}{l}
0 \\
1 \\
0
\end{array}\right]=t .
$$

The present method is said to be accurate and efficient.

\subsection{Example 2:}

The next example is a second order NDE with variable delays $\left\{t^{2},-\frac{t}{2}\right\}$

$$
\left\{\begin{array}{l}
y^{\prime \prime}(t)+y^{\prime}\left(t-t^{2}\right)-t^{2} y\left(t+\frac{t}{2}\right)+\left(y^{\prime}(t)\right)^{2}-y^{\prime}(t) y(t)=e^{t}+e^{t-t^{2}}-t^{2} e^{3 t / 2} \\
y(0)=y^{\prime}(0)=1, t \in[0,1]
\end{array}\right.
$$

The analytical solution of this problem is $y(t)=e^{t}$. We solve the problem for several values of $N$. Table 1 shows the absolute errors for $N=4,5,7$ and 9. One can see that even $N=4$ yields an accuracy up to four decimal places. Increasing $N$ decreases the absolute error for each collocation point.

Table 1. Absolute errors for several values of $N$

\begin{tabular}{|l|l|l|l|l|}
\hline$t_{i}$ & $E_{4}\left(t_{i}\right)$ & $E_{5}\left(t_{i}\right)$ & $E_{7}\left(t_{i}\right)$ & $E_{9}\left(t_{i}\right)$ \\
\hline 0.2 & $5.12 e-06$ & $2.05 e-06$ & $2.79 e-09$ & $1.86 e-12$ \\
\hline 0.4 & $2.44 e-05$ & $6.22 e-06$ & $5.53 e-09$ & $3.56 e-12$ \\
\hline 0.6 & $1.77 e-04$ & $1.37 e-05$ & $8.75 e-09$ & $4.95 e-12$ \\
\hline 0.8 & $2.08 e-04$ & $1.85 e-05$ & $4.84 e-09$ & $8.55 e-11$ \\
\hline 1.0 & $9.90 e-04$ & $9.09 e-05$ & $5.35 e-07$ & $4.78 e-09$ \\
\hline
\end{tabular}

Table 2 presents the convergence rate calculated by, [33]

$$
R_{N}=\log \left[\frac{\left\|y(t)-y_{N}(t)\right\|}{\left\|y(t)-y_{N+1}(t)\right\|}\right] \frac{1}{\log 2}, t \in[a, b] .
$$

We observe a cubic convergence for several values of $N$ at the point $t=1$.

Table 2. Convergence rate of the present method at $t=1$.

\begin{tabular}{cccccc}
\hline$N$ & 4 & 5 & 6 & 7 & 8 \\
\hline$R_{N}$ & 3.4446 & 3.5060 & 3.9020 & 3.7218 & 3.0843 \\
\hline
\end{tabular}

Figure 1 presents the analytical and approximate solution when $N=4$. One can see that the approximate solution agrees very well with the exact solution. 


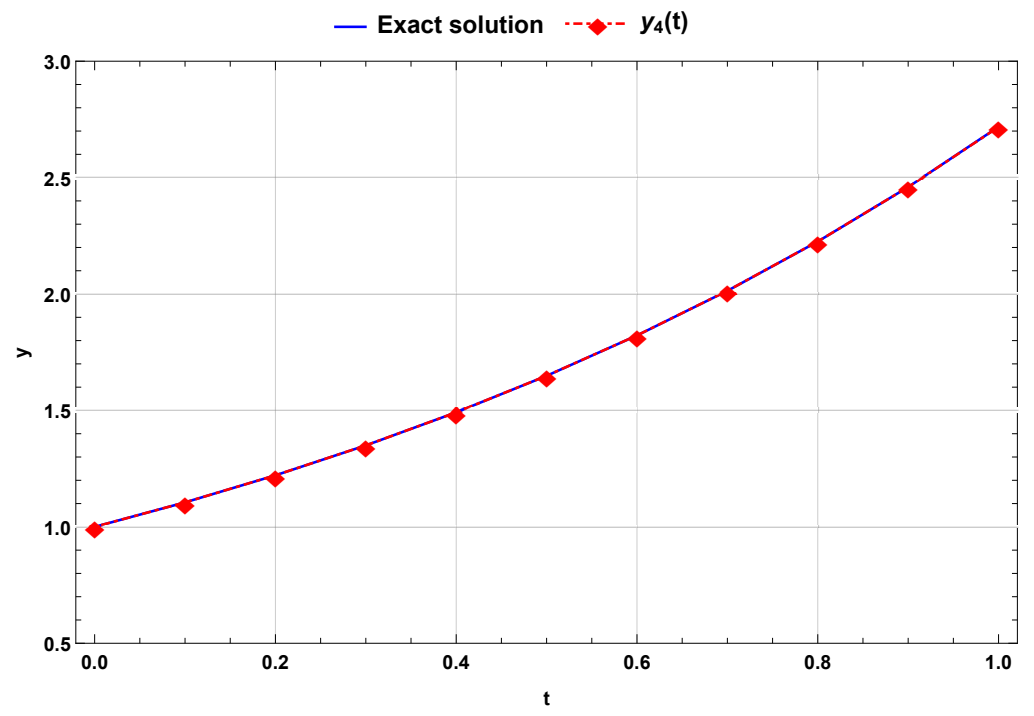

Figure 1. Exact solution and approximate solution for $N=4$.

\subsection{Example 3:}

For the third example, we consider a second order NDE with variable delay $t-t^{3} / 8$, $[10]$

$$
\left\{\begin{array}{l}
y^{\prime \prime}(t)+2 y(t)-y^{2}(t)+y\left(\frac{t^{3}}{8}\right)=\sin t-\sin ^{2} t+\sin \left(t^{3} / 8\right), \quad 0 \leq t \leq 1 \\
y(0)=0, y^{\prime}(0)=1
\end{array}\right.
$$

The analytical solution of this problem is $y(t)=\sin t$. Figure 2 presents the the exact and approximate solutions for $N=9,10$ and 11 . One can see that the numerical solution with $N=11$ agrees well with the exact solution.

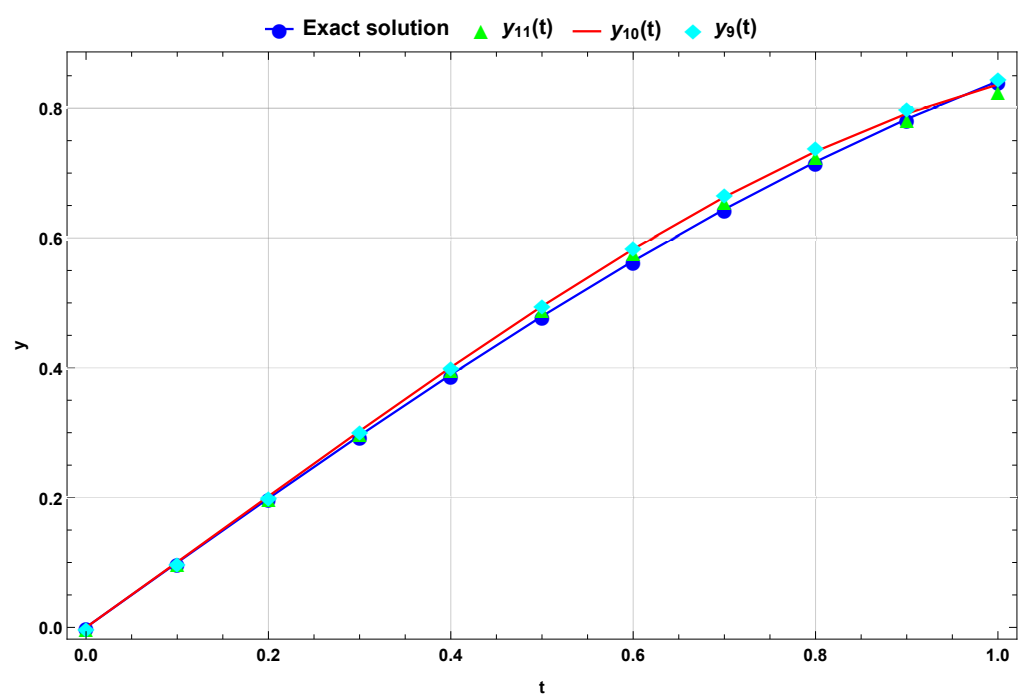

Figure 2. Comparison of analytical and numerical solutions for several values of $N$.

\section{Conclusion}

In this paper, nonlinear differential equations with variable delays are solved by Lucas polynomial approach. The main advantage of the method is to convert the nonlinear 
equations to a system of nonlinear algebraic equations. The efficiency of the proposed method is tested on three problems. The results are presented in terms of absolute errors calculated at each collocation point. It is observed that the method enables high accuracy numerical solutions or even analytical solution. Thus, we can say that this is an effective and convenient approach to solve the indicated type of problems.

\section{References}

[1] W.M. Abd-Elhameed and Y.H. Youssri, A novel operational matrix of Caputo fractional derivatives of Fibonacci polynomials: Spectral solutions of fractional differential equations, Entropy, 18 (345), 2016.

[2] W.M. Abd-Elhameed and Y.H. Youssri, Spectral solutions for fractional differential equations via a novel Lucas operational matrix of fractional derivatives, Rom. J. Phys. 61 (5-6), 795-813, 2016.

[3] W.M. Abd-Elhameed and Y.H. Youssri, Generalized Lucas polynomial sequence approach for fractional differential equations, Nonlinear Dyn. 89, 1341-1355, 2017.

[4] W.M. Abd-Elhameed and Y.H. Youssri, Spectral tau algorithm for certain coupled system of fractional differential equations via generalized Fibonacci polynomial sequence, Iran J. Sci. Technol. A, doi.org/10.1007/s40995-017-0420-9, 2017.

[5] K.S. Aboodh, R.A. Farah, I.A. Almardy and A.K. Osman, Solving delay differential equations by Aboodh transformation method, Int. J. Appl. Math. Stats. Sci. 7 (1), 21-30, 2018.

[6] I. Ali, H. Brunner, and T. Tang, A spectral method for pantograph-type delay differential equations and its convergence analysis, J. Comp. Math. 27 (2-3), 254-265, 2009.

[7] A. Ardjouni and A. Djoudi, Fixed points and stability in linear neutral differential equations with variable delays, Nonlinear Anal. 74, 2062-2070, 2011.

[8] A.G. Atta, G.M. Moatimid, and Y.H. Youssri, Generalized Fibonacci operational collocation approach for fractional initial value problems, Int. J. Appl. Comput. Math 5 (9), 2019.

[9] N. Baykus-Savaşaneril and M. Sezer, Hybrid Taylor-Lucas collocation method for numerical solution of high-order Pantograph type delay differential equations with variables delays, Appl. Math. Inf. Sci. 11 (6), 1795-1801, 2017.

[10] B. Benhammouda and H. Vazquez-Leal, A new multi-step technique with differential transform method for analytical solution of some nonlinear variable delay differential equations, SpringerPlus 5 (1723), 2016.

[11] B. Benhammouda, H. Vazquez-Leal and L. Hernandez-Martinez, Procedure for exact solutions of nonlinear pantograph delay differential equations, British J. Math. Comp. Sci. 40 (19), 2738-2751, 2014.

[12] L. Blanco-Cocom, A.G. Estrella and E. Avila-Vales, Solving delay differential systems with history functions by the Adomian decomposition method, Appl. Math. Comput. 218, 5994-6011 2012.

[13] B. Căruntu and C. Bota, Analytical approximate solutions for a general class of nonlinear delay differential equations, Scientific World J. 2014, (6 pp), 2014.

[14] G. Chen, L. Dingshi, O.V. Gaans and S.V. Lunel, Stability of nonlinear Neutral delay differential equations with variable delays, Electron. J. Differ. Eq. 2017 (118), 1-14, 2017.

[15] M. Çetin, M. Sezer and C. Güler, Lucas polynomial approach for system of high-order linear differential equations and residual error estimation, Math. Prob. Eng. 2015, (14 pp), 2015.

[16] S. Davaeifar and J. Rashidinia, Solution of a system of delay differential equations of multipantograph type, J. Taibah Univ. Sci. 11, 1141-1157, 2017. 
[17] L. Ding, X. Li, and Z. Li, Fixed points and stability in nonlinear equations with variable delays, Fixed Point Theory Appl. 2010, (14pp), 2010.

[18] J.G. Dix, Asymptotic behavior of solutions to a first-order differential equation with variable delays, Comp. Math. Appl. 50, 1791-1800, 2005.

[19] B. Dorociaková and R. Olach, Some notes to existence and stability of the positive periodic solutions for a delayed nonlinear differential equations, Open Math. 14, 361369, 2016.

[20] S. Gümgüm, N. Baykuş-Savaşaneril, Ö.,K. Kürkçü and M. Sezer, A numerical technique based on Lucas polynomials together with standard and Chebyshev-Lobatto collocation points for solving functional integro-differential equations involving variable delays, Sakarya Univ. J. Sci. 22 (6), 2018.

[21] P. Ha, Analysis and numerical solutions of delay differential-slgebraic equations, $\mathrm{PhD}$ Thesis, Berlin, 4-9, 2015.

[22] F. Ismail, R.A. Al-Khasawneh, A.S. Lwin and M. Suleiman, Numerical treatment of delay differential equations by Runge-Kutta method using Hermite interpolation, Matematika 18 (2), 79-90, 2002.

[23] C. Jin and J. Luo, Fixed points and stability in neutral differential equations with variable delay, Proc. Amer. Math. Soc. 136, 909-918, 2008.

[24] S. Karimi-Vanani and A. Aminataei, On the numerical solution of nonlinear delay differential equations, J. Concrete Applicable Math. 8 (4), 568-576, 2010.

[25] M.M. Khader, Numerical and theoretical treatment for solving linear and nonlinear delay differential equations using variational iteration method, Arab J. Math. Sci. 19 (2), 243-256, 2013.

[26] E. Lucas, Theorie de fonctions numeriques simplement periodiques, Amer. J. Math. 1, 184-240; 289-321, 1878.

[27] J.A. Martín and O. García, Variable multistep methods for delay differential equations, Math. Comput. Model. 35, 241-257, 2002.

[28] F. Mirzaee and L. Latifi, Numerical solution of delay differential equations by differential transform method, J. Sci. I. A. U. 20 (78/2), 83-88, 2011.

[29] S.T. Mohyud-Dina and A. Ylldırım, Variational iteration method for delay differential equations using He's polynomials, Z. Naturforsch. 65a, 1045-1048, 2010.

[30] H.J. Oberle and H.J. Pesch, Numerical treatment of delay differential equations by Hermite interpolation, Numer. Math. 37, 235-255, 1981.

[31] F.O. Ogunfiditimi, Numerical solution of delay differential equations using the Adomian decomposition method, Int. J. Eng. Sci. 4 (5), 18-23, 2015.

[32] M. Özel, M. Tarakçı and M. Sezer, A numerical approach for a nonhomogeneous differential equation with variable delays, Math. Sci. 12, 145-155, 2018.

[33] L. Pezza and F. Pitolli, A multiscale collocation method for fractional differential problems, Math. Comp. Simul. 174, 210-219, 2018.

[34] A.S.V. Ravi-Kanth and P. Murali-Mohan Kumar, A numerical technique for solving nonlinear singularly perturbed delay differential equations, Math. Model. Anal. 23 (1), 64-78, 2018.

[35] F. Shakeri and M. Dehghan, Solution of delay differential equations via a homotopy perturbation method, Math. Comput. Model. 48, 486-498, 2008.

[36] S.C. Shiralashetti, B.S. Hoogar and S. Kumbinarasaiah, Hermite wavelet based method for the numerical solution of linear and nonlinear delay differential equations, Int. J. Eng. Sci. Math. 6 (8), 71-79, 2017.

[37] A.G. Stephen and Y. Kuang, A stage structured predator-prey model and its dependence on maturation delay and death rate, J. Math. Biol. 49, 188-200, 2004.

[38] A.G. Stephen and Y. Kuang, A delay reaction-diffusion model of the spread of bacteriophage infection, SIAM J. Appl. Math. 65 (2), 550-566, 2005. 
[39] O.A. Taiwo and O.S. Odetunde, On the numerical approximation of delay differential equations by a decomposition method, Asian J. Math. Stat. 3 (4), 237-243, 2010.

[40] Z.Q. Wang and L.L. Wang, A Legendre-Gauss collocation method for nonlinear delay differential equations, Discrete Continuous Dyn. Syst. Ser. B 13 (3), 685-708, 2010.

[41] A. Ylldirım, H. Kocak, and S. Tutkun, Reliable analysis for delay differential equations arising in mathematical biology, J. King Saud Univ. Sci. 24, 359-365, 2012.

[42] H.Y. Youssri, A new operational matrix of Caputo fractional derivatives of Fermat polynomials: an application for solving the Bagley-Torvik equation, Adv. Diff. Eq. 2017 (73), 2017.

[43] H.Y. Youssri and W.M. Abd-Elhameed, Spectral solutions for multi-term fractional initial value problems using a new Fibonacci operational matrix of fractional integration, Progr. Fract. Differ. Appl. 2 (2), 141-151, 2016.

[44] B. Zhang, Fixed points and stability in differential equations with variable delays, Nonlinear Anal. 63, 233-242, 2005. 